\title{
Description, répartition et cycle biologique de la larve d'Hydropsyche bulgaromanorum Malicky 1977 (Trichoptera, Hydropsychidae) dans la Basse Loire
}

\author{
J.Y. Lecureuilı \\ M. Chovet ${ }^{1}$ \\ M. Bournaud2 \\ H. Tachet ${ }^{2}$
}

La larve d'Hydropsyche bulgaromanorum est décrite par comparaison avec celles d' $H$. contubermalis et $H$. omatula, avec lesquelles elle a souvent été confondue. $H$. bulgaromanorum, considérée comme une espèce plutôt médioeuropéenne et, jusqu'à présent, inconnue en France, apparaît dans la Loire dès la confluence du Cher puis devient l'espèce d'Hydropsychidae dominante de la Basse Loire. $H$. bulgaromanorum caractériserait le métapotarnal. Son cycle biologique est bivoltin.

Description, distribution and life cycle of the larva of Hydropsyche bulgaromanorum Malicky 1977 (Trichoptera) In the lower Loire.

The larva of Hydropsyche bulgaromanorum is described and compared with those of $H$. contubemalis and $H$. ornatula, two species often mixed with it. $H$. bulgaromanorum, considered as a medioeuropean species was, until now, unknown in France. It occurs in the Loire River from its confluence with the Cher and becomes the dominant species in the lower Loire. So, it is actually a species of the metapotamal. Its life cycle is bivoltine.

\section{1. - Introduction}

Pendant de nombreuses années il était pratiquement impossible d'identifier sans ambiguîté les différentes espèces d'Hydropsyche du groupe guttata; ce n'est que récemment, grâce aux travaux de Kumanski et Botosaneanu (1974) et surtout de Malicky (1977) que l'on a pu enfin disposer de descriptions précises des différentes espèces du groupe.

Sur la base de ces travaux il nous a été possible, lors de l'examen de prélèvements de benthos effectués dans la Loire, d'identifier à partir de nymphes âgées des larves d'Hydropsyche bulgaromanorum. Des captures d'adultes au filet pendant la période estivale ont confirmé la présence de cette espèce

1. SRAE - Centre. Cité administrative Coligny, 131 rue du Fau. bourg Bannier, F-45042 Orléans Cedex.

2. LA 367 du CNRS, Ecologie des Eaux douces, Université de Lyon I, F-69622 Villeurbanne Cedex. dans des proportions identiques à celles trouvées dans les récoltes de larves.

Le présent travail est consacré à la description de la larve d' $H$. bulgaromanorum et donne quelques aspects de son écologie dans la Basse Loire.

\section{2. - Milieu, matériel et méthodes}

La zone étudiée comprend la fin de la Loire Moyenne et la Basse Loire sur laquelle neuf stations réparties de Tours à Nantes ont été sélectionnées (fig. 1).

Le régime hydraulique de la Loire se caractérise par de hautes eaux hivernales et printanières suivies d'une période d'étiage prononcé de juillet à fin septembre. La station limnimétrique de Montjeansur-Loire (station 6) où l'on dispose de mesures sur 113 années (Annuaire national des débits de cours d'eau, 1981) indique un débit mensuel moyen d'août 
de $261 \mathrm{~m}^{3 /} / \mathrm{s}$ tandis que celui de février est de $1520 \mathrm{~m}^{3} / \mathrm{s}$, donc une valeur 5,8 fois supérieure.

L'hydrochimie des eaux de la Loire est influencée par la nature des terrains drainés et son régime hydraulique. La minéralisation est faible, les teneurs en calcium sont comprises entre 20 et $40 \mathrm{mg} / \mathrm{litre}$. Durant l'étiage les températures élevées de l'eau (à la station 3, moyenne mensuelle de juillet 1982 : $23,2^{\circ} \mathrm{C}$, moyenne du jour le plus chaud : $25,6^{\circ} \mathrm{C}$ ) induisent un développement algal important. Pendant cette période estivale il peut être observé également à la station 3 de fortes variations nycthémérales de pH (écart de 1 U.pH, maximum 9.4) et d'oxygène dissous (écart de $9,5 \mathrm{mg} / \mathrm{l}$, maximum de $18 \mathrm{mg} / \mathrm{l})$.

Deux types d'habitats ont été prospectés : les alluvions naturelles et les blocs. Dans la Loire moyenne les alluvions naturelles dominantes sont constituées par des cailloux que les riverains nomment le " jarre ». La taille de ceux-ci, usés par le constant frottement des sables, diminue rapidement vers l'aval. Leur taille moyenne ne dépasse pas $50 \mathrm{~mm}$ à Blois et $20 \mathrm{~mm}$ à Saumur (station 4). Par la suite, dans la traversée du Massif Armoricain, le lit mineur de la Loire est presque exclusivement constitué par du sable fin (Dion 1934).

Les blocs de la Loire Moyenne et de la Basse Loire n'ont pas une origine naturelle. Ils constituent l'enrochement de la base des digues (ou levées) et des épis nécessaires au maintien d'un chenal navigable dans la Basse Loire. Leur taille varie entre 200 et $500 \mathrm{~mm}$.

Les blocs étant très favorables à l'implantation des larves et plus accessibles à l'observateur en Basse Loire ont seuls été retenus pour l'étude de la répartition longitudinale et l'abondance relative d' $H$. bulgaromanorum par rapport aux autres espèces d'Hydropsychidae de la Loire. Les neuf stations ont été prospectées au cours des trois premiers jours de juillet 1982.

L'étude de la microrépartition préférentielle des stades larvaires en fonction du type de substrat a

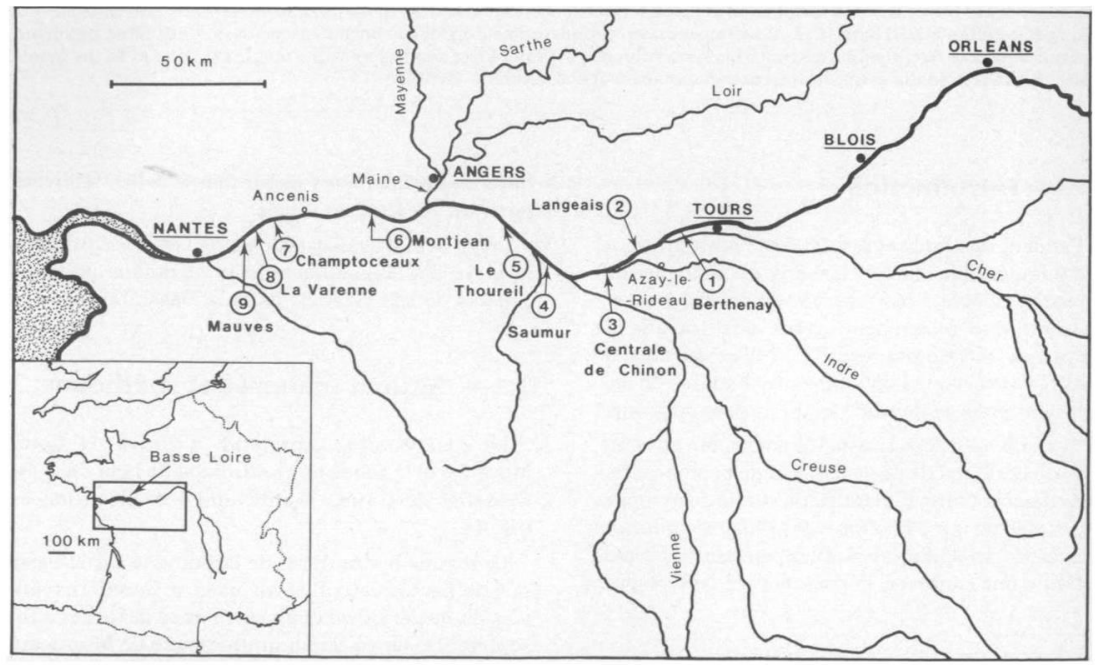

Fig. 1. - Localisation des stations d'étude (de 1 à 9) sur la fin de la Loire Moyenne et la Basse Loire (limite approximative : stations 3-4). Seuls les principaux affluents ont été figurés. 
été réalisée au niveau de la station 3 par inventaire faunistique des peuplements des blocs, d'une part, (après lavage et brossage sur tamis de $300 \mu$ ) et des zones de graviers, d'autre part, (prélèvements de $0,1 \mathrm{~m}^{2}$ de surface au moyen d'un filet Surber de $300 \mu$ de vide de maille).

La station 3, située près du site de la Centrale Nucléaire de Chinon ${ }^{1}$ est étudiée depuis plusieurs

1. Station étudiée dans le cadre d'un * suivi écologique * pour le compte d'E.D.F. années. La faune benthique a été analysée de mai à septembre 1982 à l'aide de substrats artificiels constitués de paniers en grillage métallique (maille $13 \times 13 \mathrm{~mm}$ ) de $16 \times 12,5 \times 12,5 \mathrm{~cm}$ (Khalaf et Tachet 1978) remplis de cailloux d'environ $20.30 \mathrm{~mm}$ provenant de la Loire. La durée d'immersion entre chaque récolte a été de 2 à 4 semaines. Ceci nous a permis de déterminer le cycle biologique de l'espèce. Le développement des larves d'Hydropsyche s'effectue en 5 stades. Les trois derniers stades seulement sont pris en compte ici, la détermination spécifique des larves des deux premiers stades n'étant pas possible.
Fig. 2. - Submentum (scanning) et apotome frontoclypéal. $\mathrm{a}, \mathrm{b}: H$. conturber. nalis; c. d: $H$. ornatula ; e, $\mathrm{f}: H$. bulgaromanorum. La largeur du bord antérieur de l'apotome frontoclypéal a été ramenée à une même dimension pour les 3 espèces. Voir également le texte.
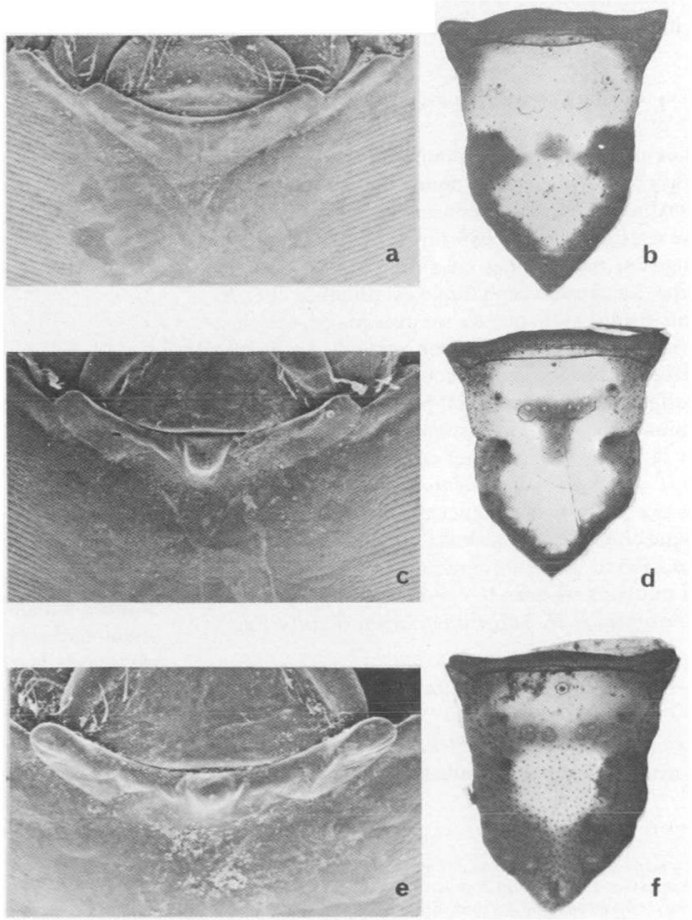


\section{3. - La larve d'H. bulgaromanorum}

La terminologie et les caractères utilisés dans cette description ont déjà été définis dans un précé. dent travail (Bournaud et al., 1982). A notre connaissance cette larve n'a pas encore été décrite. Elle ne fait pas partie des larves du groupe guttata présentées par Szczesny (1974) et cet auteur nous a confirmé, après examen de nos échantillons, que cette espèce ne lui était pas connue.

Compte tenu, comme le souligne Malicky (1977), des confusions qui ont eu lieu dans le passé, au moins au niveau des adultes, entre $H$. bulgaroma. norum, $H$. contubernalis et $H$. ornatula, nous effectuerons une description comparée des larves de ces trois espèces. La larve d'H. guttata n'ayant pas encore été identifiée il ne nous est pas possible de $l^{1}$ 'inclure dans cette comparaison ${ }^{1}$.

\section{1. - Caractères morphologiques}

Chez ces trois especes, comme chez toutes les larves du groupe guttata que nous avons pu examiner, le bord antérieur de l'apotome fronto-clypéal est convexe (fig. 2). Les prosternites postérieurs sont allongés et translucides chez ces trois espèces du groupe. La capsule céphalique est allongée chez $\boldsymbol{H}$. contubernalis alors qu'elle est presque carrée chez $H$. ornatula et $H$. bulgaromanorum (fig. 3,4 et 5), corrélativement l'apotome est court chez ces deux dernières espèces alors qu'il est plutôt allongé chez la première (fig. $2 \mathrm{~b}, \mathrm{~d}, \mathrm{f}$ ). Le submentum présente des lobes latéraux allongés chez ces trois espèces mais chez $H$. omatula et $H$. bulgaromanorum il y a toujours une petite proéminence médiane arrondie qui manque chez $\boldsymbol{H}$. contubernalis (fig. $2 \mathrm{a}, \mathrm{c}, \mathrm{e}$ ). En définitive à partir des seuls caractères morphologiques nous pouvons séparer $H$. contubernalis d'une part et $H$. omatula $-H$. bulgaromanorum d'autre part.

\section{2. - Caractères de la coloration}

Chez toutes les larves d' $H$. bulgaromanorum que nous avons pu examiner, toute la partie de l'apotome

1. La larve decrite sous le nom d' $H$. guttata par Szczesny (1974) correspond en fait à $H$. dissimulata $(=H$. modesta Navas $)$ (Szczesny, communication personnelle).

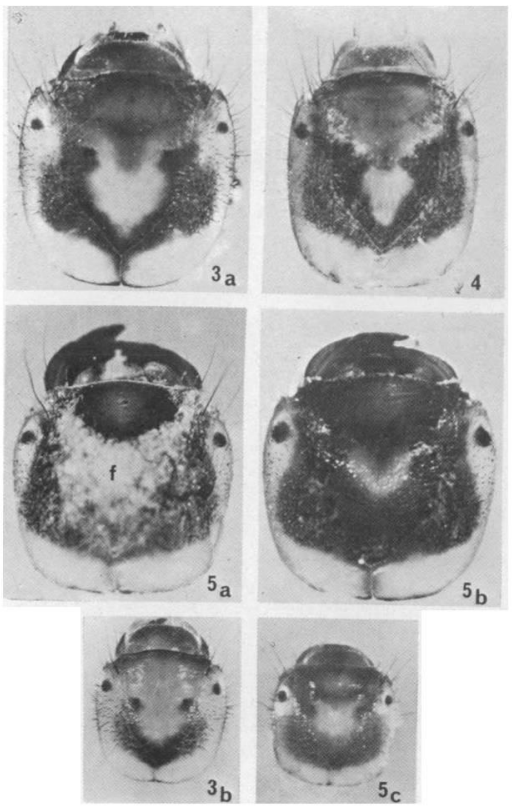

Fig. 3, 4 et 5-3: H. omatula, a : stade 5, b : stade 3. - 4: $H$. contubemalis, stade 5 . - $5: H$. bulgaromano rum, a : stade 5 normal (feutrage (f) présent), b : autre stade 5 après grattage de l'apotome, c: stade 3 .

située en arrière des muscles cibariaux est recouverte de nombreuses soies allongées, couchées et terminées par de longs et nombreux filaments fortement enchevêtrés formant ainsi un feutrage sur le dessus de la capsule céphalique (fig. $5 a$ et 6 ). Les bases d'insertion de ces soies sont bien visibles sur l'apotome isolé qui apparaît finement ponctué (fig. 2f). Si chez les larves de stades 3 et 4 et les jeunes larves de stade 5 la coloration de la cuticule reste visible, chez les larves âgées de stade 5 la coloration est presque totalement masquée, (sauf dans la partie antérieure de l'apotome qui reste dégagée) parce que de nombreux micro-organismes se développent sur ces filaments qui emprisonnent par ailleurs dans 
leurs entrelacs de fins débris d'origines diverses. Chez $H$. ornatula et chez $H$. contubernalis il y a toujours quelques soies ramifiées sur l'apotome mais celles-ci sont toujours plus courtes et ne présentent que quelques courts filaments et de ce fait chez ces deux espèces la coloration est toujours visible.

Chez les larves de stades 3 et 4 , les jeunes larves de stade 5 et après grattage de l'apotome chez les larves âgées de stade 5, la coloration de la capsule céphalique est visible. Il y a toujours une large bande sombre sur les bords de l'apotome bien développée dans la partie postérieure (fig. $2 \mathrm{f}, 5 \mathrm{~b}$ ). Cette bande qui déborde sur les pleures s'étale largement vers

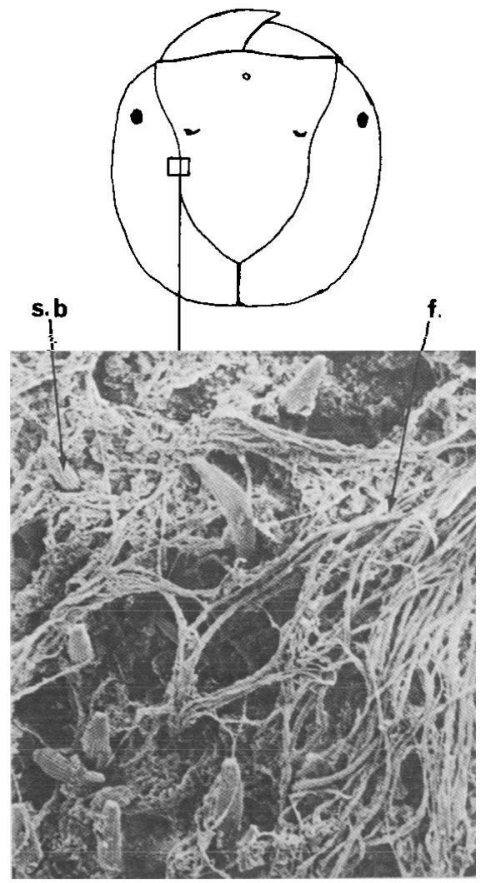

Fig. 6. - Détail du feutrage (scanning) f : filaments, s.b : sensilles basiconiques. l'arrière. Au niveau des muscles cibariaux une bande sombre transversale relie les deux bandes latérales séparant ainsi l'apotome en une partie antérieure plus ou moins claire (et dépourvue de soies) et une partie postérieure avec une tache claire médiane plus ou moins développée. Chez $H$. contubernalis l'apotome est généralement séparé en deux parties comme chez $H$. bulgaromanorum mais cette séparation se fait au niveau des prétentorinas et non au niveau des muscles cibariaux (fig. 2b). Chez $\mathrm{H}$. ornatula il n'y a qu'une étroite bande sombre le long du bord postérieur de l'apotome. La tache médiane au niveau des muscles cibariaux atteint rarement les bords de l'apotome (fig. $2 \mathrm{~d}$ ).

Les larves d' $H$. bulgaromanorum de stade 3 présentent une large bande sombre allongée transversalement, recouvrant la partie postérieure de l'apotome et débordant largement sur les pleures (fig. 5c). Chez les larves de stade $3 \mathrm{~d}^{\prime} H$. ornatula cette bande sombre est toujours plus étroite et arquée (fig. 3b).

Si les larves d' $H$. bulgaromanorum apparaissent bien différentes de celles $\mathrm{d}^{\prime} H$. ornatula et $H$. contubernalis, elles présentent, par contre, d'incontestables ressemblances avec la larve d' $H$. separata, espèce Nord-Américaine décrite par Smith (1979) : bord antérieur de l'apotome frontoclypéal convexe, tête carrée, présence d'une proéminence médiane sur le submentum, coloration de la tête très similaire. La seule différence, si elle est confirmée, concerne la structure des soies ramifiées qui couvrent la partie postérieure de l'apotome fronto-clypéal : ces soies sont courtes chez $\boldsymbol{H}$. separata et longues chez $H$. bulgaromanorum (présence d'un feutrage). Cette ressemblance est d'autant plus troublante que la structure des genitalia des mâles de ces deux espèces est très similaire (Sykora et al. 1981). De nouveaux travaux comparatifs sont nécessaires pour déterminer s'il y a ou non identité entre ces deux espèces.

\section{4. - Les larves d'H. bulgaromanorum dans la Basse Loire}

\section{1. - Répartition longitudinale}

La figure 7 donne l'abondance relative des larves d'H. bulgaromanorum inventoriées sur les blocs bordant le cours inférieur du fleuve. L'espèce est 
absente à l'amont de la confluence du Cher (station 1). A l'aval, elle est présente en proportion relativement constante ( 3 à $5 \%$ ) jusqu'à la confluence de la Vienne (stations 2 et 3). Ensuite, son abondance relative s'accroît rapidement et l'espèce devient nettement dominante sur la centaine de kilomètres à l'amont de Nantes.
Au niveau des trois dernières stations $(7,8$ et 9$)$ la Loire est soumise au régime des marées perceptibles jusqu'à Ancenis situé à $35 \mathrm{~km}$ à l'amont de Nantes. Les larves d'Hydropsychidae sont absentes du fleuve à Mauves-sur-Loire (station 9) où l'amplitude de la marée atteint un mètre. Cette amplitude est déjà de $0,5 \mathrm{~m}$ à la station 7 .

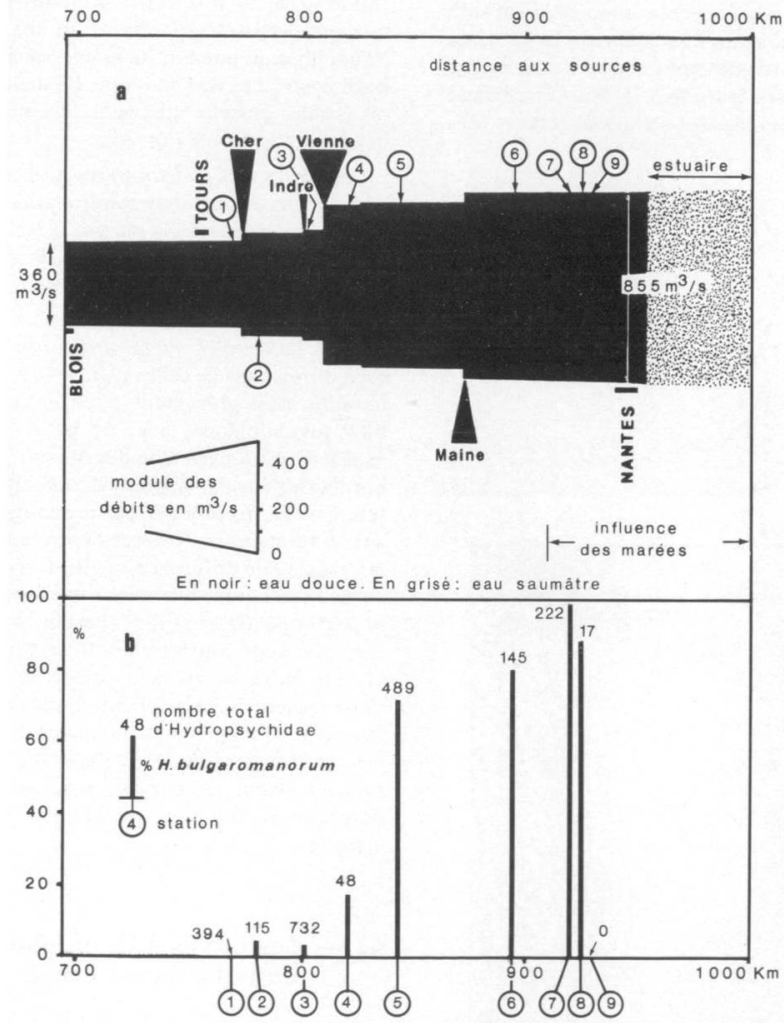

Fig. 7. - a - Profil longitudinal des débits de la Loire et de ses principaux affluents.

b - Profil longitudinal de l'abondance relative d' $H$. bulgaromanorum en pourcentages par rapport au nombre total d'Hydropsychidae indique au dessus de chaque segment.

Les nombres cerclés indiquent la position des 9 stations. 


\section{2. - Microrépartition des larves}

L'examen des échantillons prélevés sur deux habitats bien représentés au niveau de la station 3 montre une répartition préférentielle des individus de stade 3 en gravier, et de stade 5 sur les blocs (tableau I).

Tableau I : Répartition des différents stades larvaires en fonction des habitats rencontrés dans la Loire sur le site de Chinon (années 1981 et 1982 cumulées)

\begin{tabular}{lcccc}
\hline & stade 3 & stade 4 & stade 5 & $\begin{array}{c}\text { effectif } \\
\text { total }\end{array}$ \\
\hline GRAVIER & $62 \%$ & $26 \%$ & $11 \%$ & 64 \\
BLOCS & $21 \%$ & $36 \%$ & $42 \%$ & 99 \\
\hline
\end{tabular}

\section{3. - Cycle biologique}

Le cycle biologique a été étudié au niveau de la centrale nucléaire de Chinon (station 3). Les trois derniers stades larvaires ont été répertoriés sur les huit relevés de substrats artificiels échelonnés de mi-mai à fin septembre 1982 .

La figure 8a montre une abondance relative marquée des individus de stade 3 au début du mois de juin et en septembre, celle du stade 4 dans la seconde quinzaine de juin et fin septembre. Les individus de stade 5 sont dominants en mai, de fin juillet à miaoût et vraisemblablement en octobre-novembre.

L'utilisation des substrats artificiels permet d'appréhender l'évolution de la densité de population de certaines espèces en une même station. La figure $8 \mathrm{~b}$ montre la faiblesse relative des effectifs larvaires au mois de mai. La population s'accroît de manière spectaculaire au milieu du mois de juin et reste importante jusqu'à la mi-août. Les effectifs augmentent à nouveau à la fin du mois de septembre.

Ainsi, il est possible d'individualiser deux cohortes ou deux générations successives d'H. bulgaromanorum dans la Basse Loire à partir de la succession des dominances des stades larvaires (fig. 8a) et de la densité des populations ( $f \mathrm{ig} .8 \mathrm{~b}$ ) : une génération hivernale ou printanière dont le dernier stade est dominant de fin juillet à mi-août.

\section{5. - Discussion}

H. bulgaromanorum est considérée comme une espèce médio-européenne surtout répandue en
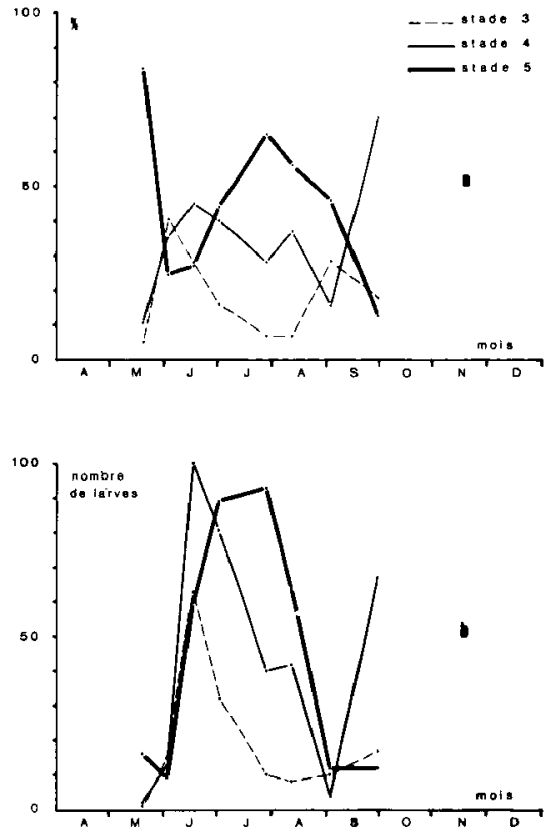

Fig. 8. - Evolution de la répartition des 3 dermiers stades larvaires $d$ 'H. bulgaromanorum au cours de la période mai-septembre 1982 à la station 3

a - Abondance relative de chaque stade par rapport à l'effectif total lors de chaque relevé.

b - Effectifs larvaires correspondants.

Europe Orientale comme le confirme les travaux de Kumanski et Botosaneanu (1974), Malicky (1977), Tobias et Tobias (1981) et Ujhelhyi (1982). Cette espèce est effectivement absente de la péninsule Ibérique (Garcia del Jalon, 1981 et Whytton Da Terra 1981) et du Maroc (Dakki 1978). Nous avons cependant trouvé des larves d' $H$. bulgaromanorum dans le Pô de Ferrare (Ghetti 1979). Du fait de cette répartition plutôt orientale, sa présence dans la Basse Loire peut paraître surprenante bien que cette espèce ait été notée en Finlande et autrefois en Angleterre (Botosaneanu et Malicky 1978). 
Malicky (1977) considère $H$. bulgaromanorum comme une espèce du métapotamal dans la mesure où, si elle représente $2 \%$ des populations d'Hydropsyche du Danube à Linz (Malicky 1981), elle devient dominante dans ce même fleuve du Défilé de Kazan jusqu'au delta (Kumanski et Botosaneanu, 1974).

Cette espèce rencontrée dans la Loire à partir de la confluence du Cher est absente de cet affluent et, d'après nos premières observations, de la Vienne. En revanche elle est présente dans l'Indre à partir d'Azay-le-Rideau (fig. 1) dans la proportion de $4 \%$ environ. Elle devient rapidement dominante dans le fleuve après les confluences qui doublent son débit, elle serait ainsi également caractéristique du métapotamal de la Loire dont la pente moyenne est inférieure à $0,2 \%$. Les caractéristiques écologiques de la Basse Loire dont la limite amont peut être fixée à la confluence de la Vienne seront précisées ultérieurement.

Dans la partie du fleuve influencée par les marées, l'espèce représente la quasi totalité des larves d'Hydropsychidae rencon trées (station 7). L'absence de cette famille dans le cours aval où l'amplitude des marées atteint et dépasse un mètre (à partir de la station 9) n'a pas permis l'étude des populations jusqu'à la limite de salinité.

H. bulgaromanorum a un cycle apparemment bivoltin dans la Basse Loire, avec une génération printanière et une génération estivale plus importante. Les individus de dernier stade larvaire sont dominants respectivement au printemps (mai) et en août. Ce cycle biologique est tout à fait semblable à celui observé sur le Haut-Rhône à Lyon pour $\boldsymbol{H}$. contu . bernalis tandis que $H$. ornatula, plus proche morphologiquement d'H. bulgaromanorum, est monovoltine (Tachet et Bournaud 1981). H. omatula est extrêmement rare dans la Loire. Elle est plus fréquente, mais sans jamais être dominante, dans le HautRhône dont le débit est de même ordre mais plus régulier, l'eau plus fraîche et la pente plus forte que dans la Basse Loire (Bournaud et al. 1982). De nouvelles investigations sont nécessaires pour préciser la position écologique d' $H$. bulgaromanorum par rapport aux autres espèces d'Hydropsychidae du bassin de la Loire qui constitue de par son originalité un cadre exceptionnel pour l'étude des populations de ces Trichoptères. Ultérieurement la comparaison avec les populations d'Hydropsychidae du bassin du Rhône, qui constitue un hydrosystème très différent, permettra une meilleure définition du profil écologique de chaque espèce.

\section{Travaux clté:}

Annuaire National des débits de cours d'eau. 1981 . - Année 1978. Vol. II : Bassin Loire-Bretagne. Imprimerie Nationale, Paris. Botosaneanu (L.) et Malicky (H.), 1978. - Trichoptera. in : Illies (J.) (ed.). Limnofauna Europeea, Stuttgart. Fischer : 333-359.

Bournaud (M.), Tachet (H.) et Perrin (J.F.). 1982. - Les Hydropsychidae (Trichoptera) du Haut-Rhône entre Genève et Lyon. Annis Limnol., $18: 61.80$.

Dakki (M.). 1978. - Le genre Hydropsyche au Maroc (Trichoptera, Hydropsychidae). Bull. Inst. Scient. Rabat, 3:111-120.

Dion (R.). 1934. - Le Val de Loire. Etude de Géographie régionale. Laffite reprints, Marseille 1978.752 p.

Garcia de Jalon (D.). 1981. - Description of Hydropsyche larvae found in the Iberian Peninsula. Proc. 3rd. intern. Symp. Trichoptera, Perugia, Junk, The Hague: 87-92.

Ghetti (P.F.). 1979. - Biological water assessment methods. Torrente Parma, Torrente Stirone, Fiume Po. 3rd technical seminar, C.E.E. : 164-169.

Khalaf (G.) et Tachet (H.). 1977. La dynamique de colonisation des substrats artificiels par les macroinvertébrés d'un cours d'eau. Annis Limnol., 13 : 169-190.

Kumanski (K.) et Botosaneanu (L.). 1974. - Les Hydropsyche du groupe guttata en Bulgarie et en Roumanie. Acta Mus. maced. Sci. mat., $14: 25-43$

Malicky (H.). 1977. - Ein Beitrag zur Kenntnis der Hydropsyche guttata - Gruppe (Trichoptera, Hydropsychidae). Z. Arbgem öst. Ent. $29: 1-28$.

Malicky (H.). 1981. - Der Indikatorwert von Köcherfliegen (Trichoptera) in großen Flüssen. Mitt. dtsch. Ges. allg. angew. Ent., 3: 135-137.

Smith (D.). 1979. - The larval stage of Hydropsyche separara Banks (Trichoptera: Hydropsychidae). Pam Pacif. Ent., 55: 10-20.

Sykora (J.L.), Swegman (B.G.) et Weawer III (J.S.). 1981. Occurence of the genus Hydropsyche in The North American great lakes. Proc. 3rd intem. Symp. Trichoptera, Perugia, Junk, The Hague : 337-345.

Szcesny (B.). 1974. - Larvae of the genus Hydropsyche (Insecta : Trichoptera) from Poland. Pol. Arch. Hydrobiol. $21: 387-390$.

Tachet (H.) et Bournaud (M.). 1981 - - Cycle biologique des Hydropsychidae et d'un Polycentropodidae du Rhône. Proc. 3rd intern. Symp. Trichoptera, Perugia, Junk, The Hague : 347.364.

Tobias (W.) et Tobias (D.). 1981. - Trichoptera germanica. Cour. Forsch. Inst. Senckenberg, $49: 1-671$

Ujhelyi (S.). 1981. - Ein Beitrag zur Verbreitung der Hydropsyche Arten (Trichoptera) in Ungaren. Folia ent. hung. $43: 191-203$.

Whytton Da Terra (L.). 1981. - Lista faunistica de Tricopteros de Portugal. Bolm. Soc. port. Ent, $12-1-42$. 